$01,10,12$

\title{
Метод построения смещенного потенциала для гипердинамического моделирования атомных систем
}

\author{
(C) Е.В. Дуда, Г.В. Корнич \\ Запорожский национальный технический университет, \\ Запорожье, Украина \\ ฯ E-mail: gkornich@zntu.edu.ua \\ (Поступила в Редакцию 21 ноября 2016 г. \\ В окончательной редакции 26 февраля 2017 г.)
}

\begin{abstract}
Рассмотрен подход к построению смещенного потенциала для гипердинамического моделирования атомных систем. С использованием данного подхода выполнено моделирование диффузии атома, адсорбированного на поверхности двумерного кристалла, и вакансии, находящейся в его объеме. Обсуждается влияние изменения потенциальных барьеров вследствие тепловых колебаний атомов на результаты расчетов. Показано, что описанное смещение потенциала в рамках гипердинамического моделирования позволяет получать статистические выборки переходов атомных систем между состояниями сходные с теми, которые дает классическая молекулярная динамика. При этом гипердинамика демонстрирует значительное вычислительное ускорение моделируемого времени по сравнению с молекулярной динамикой в случае температурноактивируемых переходов и связанных с ними процессов в атомных системах.
\end{abstract}

DOI: $10.21883 /$ FTT.2017.10.44953.418

\section{1. Введение}

Методы ускоренной молекулярной динамики были предложены как способы динамического моделирования на временны́х промежутках, недостижимых для метода классической молекулярной динамики (МД). К таким методам относятся температурно-ускоренная динамика [1], гипердинамика (ГД) [2], метод параллельных реплик $[3,4]$. Необходимость использования этих методов возникает при изучении процессов, в которых доминируют, так называемые, редкие события - температурно-активируемые переходы атомных систем между состояниями с разной локализацией атомов. Практическими приложениями этих методов могут быть модельные представления диффузии, структурной перестройки поверхности, образования дефектов и других явлений, которые классическая МД не имеет возможности рассматривать на характерных для таких явлений, микросекунды и более, промежутках времени. Достоинством методов ускоренной МД перед альтернативными методами, такими как кинетический Монте-Карло [5], является отсутствие необходимости составления предварительного списка возможных элементарных событий. В данных методах физические механизмы проявляются сами при решении классических уравнений движения атомов.

Методы ускоренной МД уже успешно использовались для исследования различных атомных систем и фрагментов устройств, таких как кристаллы [6-8], биомолекулы [9-11], рабочая часть зонда АСМ вблизи поверхности [12]. Эти методы представляют интерес для атомистического моделирования процессов, сопровождающих осаждение и приводящих к образованию различных эпитаксиальных наноструктур. В частности, массоперенос по грани нановискера [13]; самоорганизация новых, толщиной в один атомный слой, аллотропных форм традиционных материалов электроники на известных подложках $[14,15]$ и наноустройств на их основе. Методы ускоренной МД, в принципе, позволяют пошагово достигать интервалов времени, необходимых для формирования модельных фрагментов соответствующих атомных структур.

В настоящей работе рассмотрен гипердинамический подход, который заключается в увеличении частоты переходов системы посредством понижения энергетических барьеров между различными состояниями атомных систем. Рассмотрен способ понижения энергетических барьеров, который является достаточно универсальным. Было проведено гипердинамическое моделирование диффузии адсорбированного атома и вакансии на примере двумерной атомной решетки с использованием предложенного способа понижения энергетических барьеров, а также выполнено сравнение полученных результатов с результатами МД-моделирования для оценки адекватности работы гипердинамического подхода.

\section{2. Формулировка метода}

При переходе между минимумами потенциальной энергии атом проходит через область, являющуюся окрестностью седловой точки. Константа скорости для такого перехода, согласно гармоническому приближению теории переходного состояния [16], может быть определена из следующего выражения:

$$
k=\frac{\prod_{i}^{3 N} v_{i}^{\text {init }}}{\prod_{i}^{3 N-1} v_{i}^{*}} \exp \left[-\left(E^{*}-E^{\text {init }}\right) / k_{\mathrm{B}} T\right],
$$

где $T-$ температура системы; $k_{\mathrm{B}}-$ постоянная Больцмана; $N-$ количество атомов в системе; $E^{*}-$ энергия седловой точки; $E^{\text {init }}-$ энергия локального 
энергетического минимума, соответствующего начальному положению атома; $v_{i}^{*}$ - частоты нормальных мод колебаний, соответствующие положению системы в переходном состоянии; $v_{i}^{\text {init }}-$ частоты нормальных мод колебаний для системы в начальном состоянии. Разность энергий $E^{\text {act }}=E^{*}-E^{\text {init }}$ в уравнении (1) является энергией активации перехода.

Рассмотрим две системы, для которых всякому переходу в новое состояние системы I можно поставить в соответствие переход системы II, который будет иметь меньшую на некоторую величину $\Delta E$ энергию активации, а также предэкспоненциальные множители в выражении (1) для всех соответствующих переходов этих систем будут одинаковы. Далее будем называть соответствующими аналогичные характеристики систем I и II для поставленных в соответствие переходов. Отношение констант скоростей для соответствующих переходов таких систем согласно (1) имеет вид

$$
k_{\mathrm{II}} / k_{\mathrm{I}}=\exp \left(\Delta E / k_{\mathrm{B}} T\right) .
$$

Отношения же констант скоростей любых двух переходов $\alpha$ и $\beta$ системы II будут равны соответствующим отношениям для системы I. Это является справедливым в связи с тем, что энергии переходов в системе II отличаются от энергий соответствующих переходов в системе I на одинаковую величину. Следовательно, разность энергий активации любых двух переходов системы I равна разности энергий активации соответствующих переходов системы II. Кроме того, соответствующие предэкспоненциальные множители в уравнении (1) для этих систем совпадают.

Таким образом

$$
k_{\mathrm{II}, \alpha} / k_{\mathrm{II}, \beta}=k_{\mathrm{I}, \alpha} / k_{\mathrm{I}, \beta} .
$$

Из всего вышесказанного следует, что эволюцию некоторой системы, связанную с выполнением последовательности, так называемых, редких событий, можно изучать, моделируя методом молекулярной динамики соответствующую систему, имеющую меньшие энергии переходов и такие же частоты нормальных мод. Среднее время, которое будет затрачено системой I на всякий переход, совершенный системой II с пониженными энергетическими барьерами, можно определить, согласно соотношению (2), следующим образом:

$$
t_{\mathrm{II}}=t_{\mathrm{I}} \exp \left(-\Delta E / k_{\mathrm{B}} T\right)
$$

\section{3. Построение системы с пониженными энергетическими барьерами}

Пусть потенциальная энергия системы описывается функцией $U\left(r_{1}, r_{2}, \ldots, r_{N}\right)$. Система с потенциальной энергией $U^{\prime}\left(r_{1}, r_{2}, \ldots, r_{N}\right)$, согласно выражению (5), имеет пониженные на одинаковую величину энергии

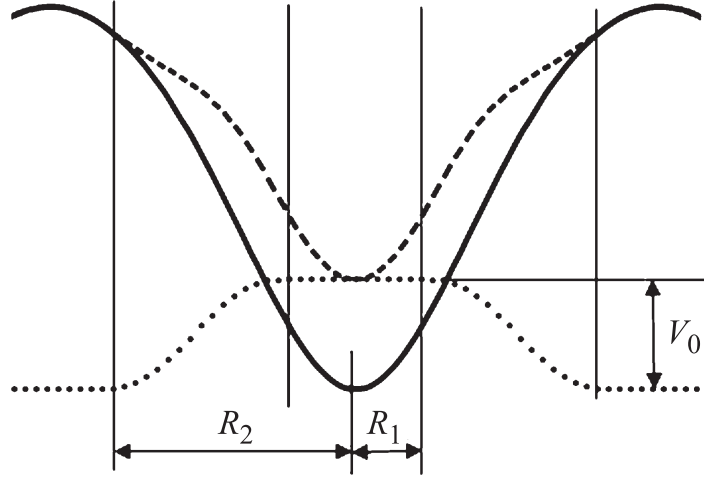

Рис. 1. Сечения потенциальных энергетических ям, образованных оригинальным (сплошная линия) и измененным (штриховая линия) потенциалами. Пунктирная линия представляет функцию $V\left(\rho_{i}\right)$. Вертикальные линии расположены на расстояниях $R_{1}$ и $R_{2}$ от минимума.

активации всех переходов, но такую же форму поверхности потенциальной энергии в окрестностях всех энергетических минимумов и седловых точек

$$
U^{\prime}\left(r_{1}, r_{2}, \ldots, r_{N}\right)=U\left(r_{1}, r_{2}, \ldots, r_{N}\right)+\sum_{i}^{N} V\left(\rho_{i}\right),
$$

где $\rho_{i}-$ расстояние от $i$-го атома до ближайшего энергетического минимума. Функция $V\left(\rho_{i}\right)$ имеет следующий вид:

$$
V\left(\rho_{i}\right)= \begin{cases}V_{0}, & \rho_{i}<R_{1} \\ \varphi\left(\rho_{i}\right), & R_{1} \leq \rho_{i}<R_{2}, \\ 0, & \rho_{i} \geq R_{2}\end{cases}
$$

где $R_{1}, R_{2}, V_{0}$ - заданные параметры; $\varphi\left(\rho_{i}\right)$ - некоторая функция, обеспечивающая непрерывность $V\left(\rho_{i}\right)$ и некоторого наперед заданного числа $n$ ее производных

$$
\left\{\begin{array}{l}
\varphi\left(R_{1}\right)=V_{0} \\
\varphi\left(R_{2}\right)=0 \\
\varphi^{(m)}\left(R_{1}\right)=0 \\
\varphi^{(m)}\left(R_{2}\right)=0
\end{array},\right.
$$

где $m=1,2, \ldots, n$.

Функция $\varphi\left(\rho_{i}\right)$ может быть представлена, например, в виде полинома степени $2 n+1$ или в виде сплайна. Таким образом, в окрестности каждого энергетического минимума потенциал взаимодействия изменяется так, как показано на рис. 1. Потенциал, качественно близкий к выражению (6), был так же использован в работе [12] для описания взаимодействия закругления зонда АCM с подложкой в условиях низких температур.

Из рис. 1 видно, что потенциал $V\left(\rho_{i}\right)$ имеет такой вид, что значения производных функции $U^{\prime}\left(r_{1}, r_{2}, \ldots, r_{N}\right)$ отличаются от соответствующих значений производных функции $U\left(r_{1}, r_{2}, \ldots, r_{N}\right)$ только в пределах расстояний больших $R_{1}$ и меньших $R_{2}$ от минимумов данных 
функций. Эти расстояния следует подбирать так, чтобы частоты нормальных мод систем, чьи потенциальные энергии описываются функциями $U^{\prime}\left(r_{1}, r_{2}, \ldots, r_{N}\right)$ и $U\left(r_{1}, r_{2}, \ldots, r_{N}\right)$, в окрестности энергетических минимумов и седловых точек совпадали. Это можно достичь, подбирая $R_{1}$ так, чтобы значение энергии в любой точке, находящейся от минимума энергии на этом расстоянии, отличалось от значения энергии минимума на величину, большую средней энергии атомов при данной температуре. Тогда отличия в значениях производных между оригинальным и измененным потенциалами на интервале от $R_{1}$ до $R_{2}$ не будут сказываться на значении нормальных мод колебаний. Энергии всех потенциальных минимумов при такой модификации измененного потенциала меняются на одинаковую заданную величину $\mathrm{V}_{0}$.

При построении измененного потенциала следует учесть, что во время тепловых колебаний атомов поверхность потенциальной энергии меняется. С учетом этого, оптимально подобрать параметры дополнительного потенциала $V\left(\rho_{i}\right)$ можно, например, исследовав оригинальную систему на некотором промежутке времени МД-методом. Таким способом можно получить значения некоторых критически важных параметров, а именно: разности энергий в точках минимумов и точках, находящихся на расстоянии $R_{1}$ от них; разности энергий точек минимумов и седловых точек; расстояния между точками минимумов и седловыми точками. Значения $R_{1}$, $R_{2}, V_{0}$ следует подбирать так, чтобы не появлялись энергетические минимумы, которых нет в оригинальной системе. Расстояние $R_{2}$ должно всегда быть меньше расстояния между минимумом энергии и любой из седловых точек, чтобы седловые точки не смещались относительно своих положений в оригинальных системах. При этом, используя МД-метод, достаточно рассмотреть части системы, содержащие наиболее низкие энергетические барьеры, поскольку именно они ограничивают значение параметра $V_{0}$. В результате потенциальная энергия $U^{\prime}\left(r_{1}, r_{2}, \ldots, r_{N}\right)$ будет такой, что удовлетворятся выражения (2) и (3). МД-моделирование системы с такой энергией может быть использовано для изучения эволюции системы с энергией $U\left(r_{1}, r_{2}, \ldots, r_{N}\right)$, причем скорость диффузионных процессов в первой системе, согласно (4), будет в $\exp \left(V_{0} / k_{\mathrm{B}} T\right)$ раз больше, чем во второй.

Моделирование показало, что нахождение мгновенных положений энергетических минимумов является важной задачей. На рис. 2 изображены корректно построенный (пунктирная линия) и построенный на основании неверно найденного положения энергетического минимума (штриховая линия) измененные потенциалы. Во втором случае $V\left(\rho_{i}\right)$ сместился на некоторое расстояние относительно верного положения. Как видно благодаря вертикальным линиям $a$ и $b$, из-за недостаточной точности определения положения минимума, значение энергии атома в некоторых областях пространства отличается от того, какое он должен был бы иметь в случае корректного определения положения минимума. Погрешность определения координат энергетических

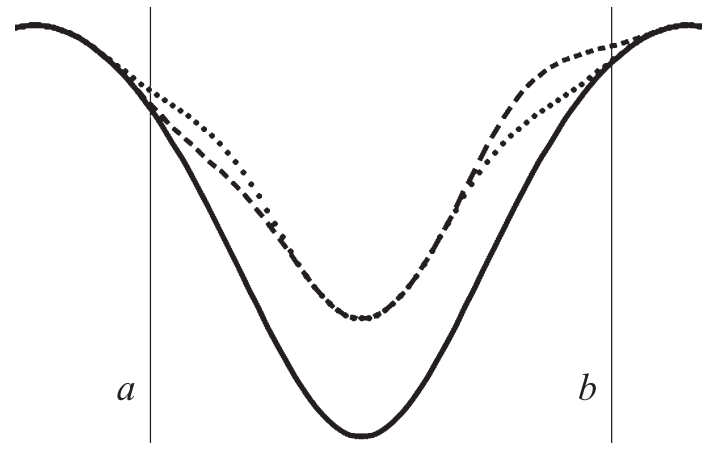

Рис. 2. Потенциальные ямы, образованные оригинальным (сплошная линия) и измененными потенциалами, а именно, яма, образованная корректно построенным измененным потенциалом (пунктирная линия) и яма, построенная на основании неверно найденного положения энергетического минимума (штриховая линия).

минимумов может приводить как к понижению, так и к повышению мгновенного значения энергии атома по сравнению с истинным значением измененного потенциала. В результате реальное смещение энергий активации может различным образом отличаться от $V_{0}$ для разных энергетических ям. Точность нахождения потенциальных минимумов должна быть такой, чтобы погрешность вычисления энергии атома в смещенном потенциале была малой по сравнению с $V_{0}$.

Для получения дополнительного ускорения вычислений, потенциал $V\left(\rho_{i}\right)$ в выражении (5) можно не добавлять вблизи тех энергетических минимумов, в окрестностях которых находятся атомы, не участвующие в переходах. В этом случае нет необходимости в нахождении соответствующих энергетических минимумов. Тогда суммирование во втором слагаемом в выражении (5) будет производиться по потенциальным ямам, в которых находятся атомы, способные осуществить переход.

Вычислительное ускорение, получаемое при ГД-моделировании, может быть оценено выражением

$$
t_{\mathrm{HD}} / t_{\mathrm{MD}}=1 /(1+\varepsilon \eta) \exp \left(V_{0} / k_{\mathrm{B}} T\right),
$$

где $\eta$ - доля атомов системы, для которых производится поиск ближайших энергетических минимумов. В правой части выражения (8) введением параметра $\varepsilon$ учитывается так же время, затрачиваемое при ГД-моделировании на определение положения энергетических минимумов. В данной работе для определения положения минимумов использовался метод градиентного спуска. Координаты минимума уточнялись до тех пор, пока ускорение атома меди, помещенного в данную точку, не становилось меньше $10^{-4} \AA / \mathrm{ps}^{2}$. В этом случае среднее значение коэффициента $\varepsilon$ было равно 3.

\section{4. Описание модели}

C целью проверки применимости ГД-метода для описания эволюции атомных систем было проведено 
моделирование с использованием этого подхода, а также метода классической МД и сравнение полученных результатов. Для получения достаточного количества событий термически активированных переходов в широком диапазоне температур, моделирование атомной системы проводилось в двумерном пространстве. Система состояла из двумерной медной подложки и адсорбированного на ее поверхности атома меди. В модели был использован потенциал Морзе (Morse) [17,18], который на расстояниях, меньше $1 \AA$, сшивался посредством полинома пятой степени с потенциалом Зиглера-Бирзака-Литтмарка (Zigler-Biersack-Littmark) [19]. Подложка с гексагональной упаковкой имела 32 атомных слоя в глубину и ширину. Четыре нижних атомных слоя были неподвижны, а на боковых сторонах были установлены периодические граничные условия. Для поддержания постоянной температуры кристалла $T_{0}$ использовался метод термической ванны Берендсена (Berendsen) [20]. Вводимая, согласно методу Берендсена, дополнительная сила действует в пределах двух атомных слоев, граничащих с неподвижными донными слоями, а также двух атомных слоев, расположенных на боковых краях подложки перпендикулярно свободной поверхности

$$
F=-0.75 v m \omega_{\mathrm{D}}\left(1-T_{0} / T\right),
$$

где $v$ - скорость атома, $m$ - масса атома; $\omega_{\mathrm{D}}$ - частота Дебая (Debye) материала подложки; $T$ - температура подложки в данный момент времени. Мгновенная температура определялась следующим образом:

$$
T=\bar{E}_{k} / k_{\mathrm{B}},
$$

где $\bar{E}_{k}-$ мгновенное значение средней кинетической энергии атомов. Выражение (10) отражает двумерный характер моделирования. Боковые атомные слои, на которые действует сила (9), вновь определялись после каждого акта перехода адсорбированного атома так, чтобы расстояние от этих слоев до него не было меньше восьми параметров решетки. Функция $\varphi\left(\rho_{i}\right)$ из выражений (6) и (7) была представлена в виде полинома пятой степени.

В каждом численном эксперименте моделирование диффузии адсорбированного на подложке атома продолжалось до накопления 800 событий переходов системы между состояниями. Далее сравнивались функции распределения времени, затраченного на переходы, полученные с помощью методов ГД и МД. Для проверки гипотезы о соответствии полученных методами ГД- и МД-распределений одному и тому же закону, использовался критерий однородности Смирнова [21].

\section{5. Результаты и их обсуждение}

Моделирование диффузии адсорбированного атома на поверхности подложки проводилось при температу$\operatorname{pax} 100,150$ и $200 \mathrm{~K}$. Для каждой температуры выполнялся один численный эксперимент методом классической МД- и три - ГД-методом с различными значениями параметров $R_{1}, R_{2}, V_{0}$. Результаты моделирования
Таблица 1. Моделирование диффузии адсорбированного атома по поверхности подложки методами МД- и ГД- при различных температурах. Каждому МД-эксперименту соответствует три ГД-эксперимента с различными значениями параметров $R_{1}, R_{2}, V_{0}$ и средним временем перехода $t$ для данной

\begin{tabular}{|c|c|c|c|c|c|c|}
\hline \multirow{2}{*}{$\begin{array}{c}T_{0}, \\
\mathrm{~K}\end{array}$} & \multicolumn{2}{|c|}{$\mathrm{t}, \mathrm{ps}$} & \multirow{2}{*}{$\begin{array}{l}V_{0} \\
\mathrm{eV}\end{array}$} & \multirow{2}{*}{$\begin{array}{c}R_{1}, \\
\AA\end{array}$} & \multirow{2}{*}{$\begin{array}{c}R_{2} \\
\AA\end{array}$} & \multirow{2}{*}{$\alpha$} \\
\hline & МД & ГД & & & & \\
\hline \multirow{3}{*}{200} & \multirow{3}{*}{142} & 148 & 0.01 & 0.25 & 0.8 & 0.1 \\
\hline & & 135 & 0.01 & 0.2 & 0.75 & 0.05 \\
\hline & & 142 & 0.015 & 0.25 & 0.8 & 0.1 \\
\hline \multirow{3}{*}{150} & \multirow{3}{*}{758} & 713 & 0.01 & 0.3 & 0.8 & 0.05 \\
\hline & & 688 & 0.012 & 0.3 & 0.8 & 0.001 \\
\hline & & 811 & 0.012 & 0.32 & 0.9 & 0.1 \\
\hline \multirow{3}{*}{100} & \multirow{3}{*}{19955} & 24194 & 0.012 & 0.32 & 0.9 & 0.01 \\
\hline & & 19359 & 0.012 & 0.25 & 0.9 & 0.1 \\
\hline & & 13480 & 0.015 & 0.25 & 0.8 & - \\
\hline
\end{tabular}
статистики экспериментов

представлены в табл. 1. Гипотеза о соответствии полученных МД- и ГД-методами эмпирических распределений одному закону распределения тестировалась критерием однородности Смирнова при следующих значениях уровня значимости $\alpha$ : $0.001,0.005,0.01,0.025,0.05$, 0.1. В последнем столбце табл. 1 указано максимальное значение $\alpha$, для которого гипотеза принимается. Знак „-“ в последнем столбце табл. 1 означает, что нулевая гипотеза о принадлежности выборок, полученных МД- и ГД-методами, одному закону распределения, отклоняется для всех рассматриваемых уровней значимости, т.е. полученные распределения критически отличаются друг от друга.

Представляет интерес, что смещенный потенциал с одинаковыми параметрами был использован при температурах 100 и $200 \mathrm{~K}$ (третья строка в табл. 1 для каждой из данных температур), однако в первом случае нулевая гипотеза была отклонена, а во втором - принята на уровне значимости $\alpha=0.1$.

На рис. 3 изображена потенциальная яма, в которой находится адсорбированный атом. Горизонтальное направление соответствует координате вдоль прямой, проходящей через энергетический минимум и седловую точку, соответствующую некоторому переходу. На рис. 3, $a$ представлен минимальный потенциальный барьер, обнаруженный при МД-моделировании системы в течение 10 ps. На рис. $3, b-$ минимальный потенциальный барьер, обнаруженный при МД-моделировании системы в течение 100 ps. Оба рисунка соответствуют одному переходу в разные моменты времени. Данные кривые получены для температуры $100 \mathrm{~K}$.

Как видно из рис. 3, $b$, при изменении потенциала, седловая точка смещается относительно своего положения в случае оригинального потенциала. В результате имеет место изменение энергии перехода, отличное от наперед заданного значения $V_{0}$. Также изменены 

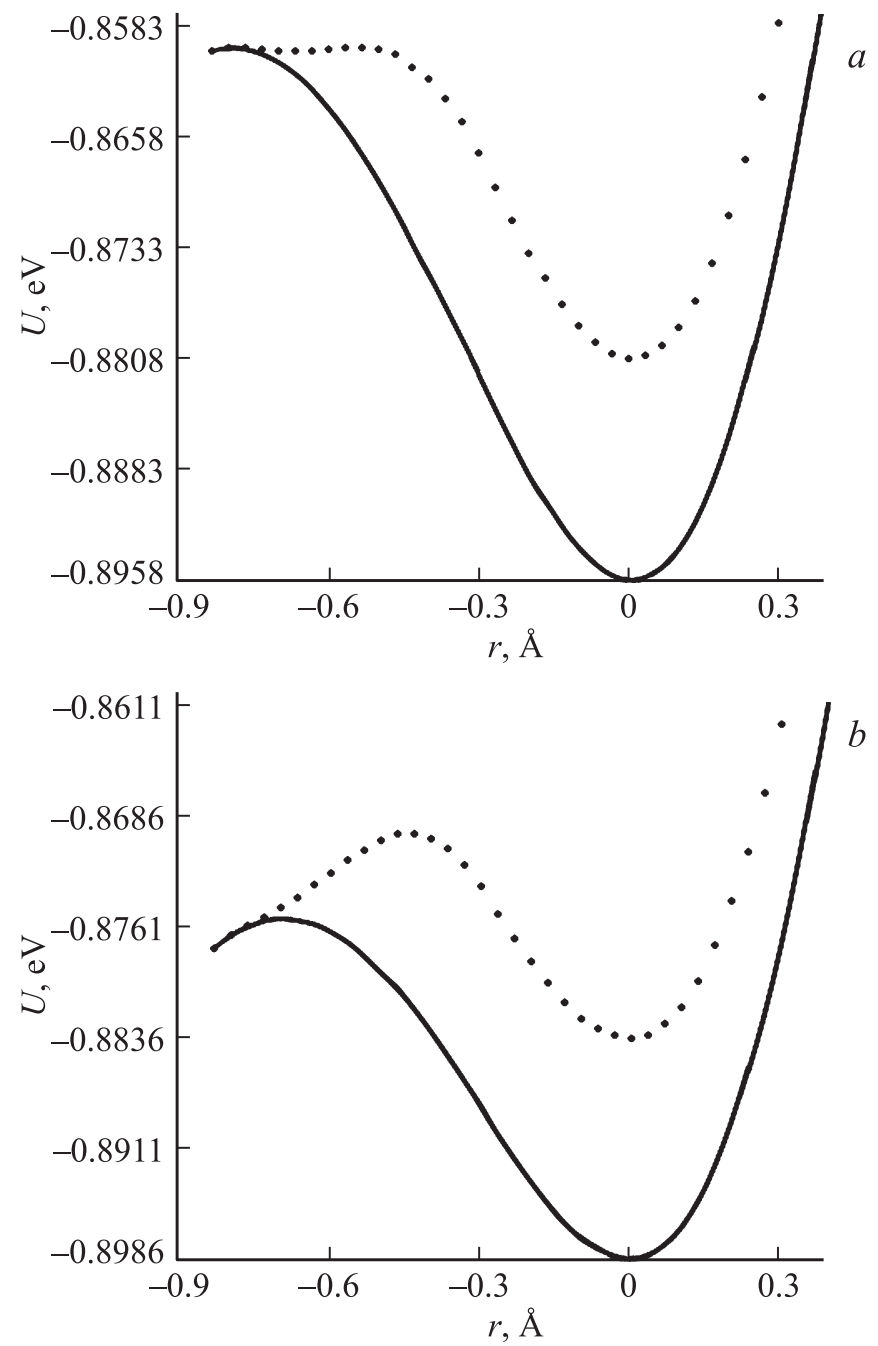

Рис. 3. Потенциальная яма (сплошная линия) в системе с оригинальным потенциалом вдоль направления, соединяющего энергетический минимум и седловую точку; яма для системы с измененным потенциалом (пунктирная линия), параметры которого $R_{1}=0.25 \AA, R_{2}=0.8 \AA, V_{0}=0.015 \mathrm{eV}$. $U-$ потенциальная энергия, $r$ - расстояние до минимума потенциальной ямы. $a$ и $b$ соответствуют одной потенциальной яме в разные моменты времени.

нормальные моды в седловой точке. Подобный эффект может объяснить несоответствия результатов, полученных ГД- и МД-методами при температуре $100 \mathrm{~K}$ для значений параметров смещенного потенциала, соответствующих последней строке табл. 1. Однако результаты ГД-моделирования при температуре $200 \mathrm{~K}$ с такими же параметрами измененного потенциала хорошо согласуются с результатами МД-моделирования. Это означает, что доля переходов через низкие барьеры, аналогичные барьеру, изображенному на рис. $3, b$, для которых измененный потенциал перестает удовлетворять необходимым условиям, невелика в этом случае. Это выглядит вполне возможным, поскольку частота переходов через барьер некоторой высоты зависит не только от константы скорости $k$ из уравнения (1), но и от частоты $p$ появления этих барьеров. Рассмотрим два состояния $\alpha$ и $\beta$ некоторого барьера, различающиеся высотой. Пусть для переходов через данный барьер выполняется неравенство

$$
k_{\alpha} p_{\alpha}>k_{\beta} p_{\beta}
$$

Неравенство (11) означает, что переходы через барьер в состоянии $\alpha$ происходят чаще, чем в состоянии $\beta$. Данное неравенство может выполняться, даже если барьер в состоянии $\beta$ имеет меньшую высоту, чем в состоянии $\alpha$. Для этого необходимо, чтобы $p_{\alpha}$ было значительно больше $p_{\beta}$. Однако при понижении температуры константа скорости $k_{\beta}$ может стать настолько больше, чем $k_{\alpha}$, что будет выполняться неравенство, обратное неравенству (11). Таким образом, с понижением температуры низкие барьеры, частота появления которых может быть небольшой, играют все большую роль, что необходимо учитывать при построении измененного потенциала.

Выполненное моделирование показало, что ГД-метод при корректном подборе параметров может давать такие же выборки переходов, которые можно получить методом классической МД. Однако в приведенной двумерной системе, в связи с малыми значениями высот энергетических барьеров для адсорбированного атома, не удается построить измененный потенциал с таким значением параметра $V_{0}$, чтобы имело место существенное ускорение по моделируемому времени.

С целью получения большего вычислительного ускорения было проведено моделирование диффузии вакансии в двумерном кристалле меди. Для этого процесса энергетические барьеры достаточно велики, чтобы можно было выбирать большие значения $V_{0}$, чем в предыдущем случае. Данная система отличалась от описанной выше системы поверхностной диффузии адсорбированного атома отсутствием неподвижных атомов, а также присутствием сверху и снизу кристалла периодических граничных условий. Диссипативные слои были установлены вблизи атомных слоев, подчиняющихся периодическим граничным условиям. С целью наглядного представления возможностей метода ГД, постоянная решетки кристалла вдоль определенного направления была искусственно уменьшена на величину $z$, а в перпендикулярном направлении - увеличена на ту же величину. Направление сжатия кристалла было выбрано таким образом, что доступные в любой момент времени шесть переходов вакансии разделились на два типа: два перехода вдоль направления, в котором было произведено сжатие, и четыре перехода в других направлениях. В каждом эксперименте было получено 800 переходов на расстояниях не меньших одной постоянной решетки от атомов диссипативных слоев. Моделирование проводилось при температурах системы $400 \mathrm{~K}$ и $500 \mathrm{~K}$. Для ГД-моделирования были выбраны следующие параметры: $R_{1}=0.3 \AA, R_{2}=0.9 \AA$. Параметр $V_{0}$ имел различные значения при различных температурах.

Результаты моделирования представлены в табл. 2. Гипотеза о соответствии полученных МД- и ГД-методами 
Таблица 2. Моделирование диффузии вакансии в объеме двумерного деформированного кристалла меди: $t$ - среднее время, проходящее между двумя последовательными переходами; $z$ - величина сжатия кристалла в некотором направлении и растяжения в перпендикулярном ему направлении; $n-$ доля переходов, совершенных вдоль направления сжатия кристалла

\begin{tabular}{|c|c|c|c|c|c|}
\hline \multirow{2}{*}{ Параметр } & \multicolumn{2}{|c|}{ МД } & \multicolumn{2}{|c|}{ ГД } & \multirow{2}{*}{$\begin{array}{l}V_{0} \\
\mathrm{eV}\end{array}$} \\
\hline & $t$, ps & $n$ & $t, \mathrm{ps}$ & $n$ & \\
\hline $\begin{array}{l}T=500 \mathrm{~K} \\
z=0.25 \%\end{array}$ & 181 & 0.433 & 180 & 0.414 & 0.08 \\
\hline $\begin{aligned} T & =400 \mathrm{~K} \\
z & =0.5 \%\end{aligned}$ & 1461 & 0.58 & 1433 & 0.485 & 0.07 \\
\hline $\begin{array}{l}T=400 \mathrm{~K} \\
z=0.25 \%\end{array}$ & 1654 & 0.453 & 1527 & 0.446 & 0.065 \\
\hline
\end{tabular}

эмпирических распределений одному закону распределения тестировалась критерием однородности Смирнова. Для всех представленных случаев гипотеза удовлетворяется при уровне значимости $\alpha=0.1$. Видно, что ГД-метод позволяет получить близкие к значениям, полученным МД-методом, не только времена переходов, но и долю переходов, осуществляемых через некоторый энергетический барьер по отношению к общему числу переходов. Результаты показали, что в случае моделирования такой системы при температурах $100-200 \mathrm{~K}$ можно получать вычислительное ускорение продолжительности моделирования на два-три порядка. В трехмерных системах подобные ускорения можно получать при больших температурах, поскольку энергетические барьеры в них существенно выше, чем в рассмотренных здесь двумерных системах.

\section{6. Заключение}

Рассмотрен способ понижения энергетического барьера при ГД-моделировании, позволяющий получать выборку с тем же законом распределения, что и МД-моделирование. Данное утверждение было проверено для системы, совершающей переход лишь одного типа (диффузия адсорбированного атома), и системы, у которой в каждый момент времени существует два типа различных конкурирующих переходов (диффузия вакансии в искусственно искаженной решетке).

Показано, что ГД-моделирование системы при различных температурах с одинаковым смещенным потенциалом может приводить к результатам, значительно отличающимся точностью. В общем случае значения параметров смещенного потенциала следует определять отдельно для каждой исследуемой температуры системы.

Используемый метод позволяет получать тем большее ускорение по времени моделирования, чем более высоким является самый низкий энергетический барьер в системе.
Авторы выражают благодарность Суперкомпьютерному комплексу МГУ им. М.В. Ломоносова [22] за предоставление высокопроизводительных вычислительных ресурсов для проведения компьютерного моделирования.

\section{Список литературы}

[1] A.F. Voter. J. Chem. Phys. 106, 11, 4665 (1997).

[2] A.F. Voter. Phys. Rev. Lett. 78, 20, 3908 (1997).

[3] A.F. Voter. Phys. Rev. B 57, 22, 985 (1998).

[4] D. Perez, B.P. Uberuaga, A.F. Voter. Comp. Mater. Sci. 100, 90 (2015).

[5] D.T. Gillespie. J. Comp. Phys. 22, 403 (1976).

[6] M. Hong, J.L. Wohlwend, R.K. Behera, S.R. Phillpot, S.B. Sinnott, B.P. Uberuaga. Surf. Sci. 617, 237 (2013).

[7] V. Georgieva, A.F. Voter, A. Bogaerts. Cryst. Growth Design 11, 6, 2553 (2011).

[8] F. Montalenti, A.F. Voter. Phys. Status Solidi B. 226, 1, 21 (2001).

[9] D. Hamelberg, J. Mongan, J.A. Mc Cammon. J. Chem. Phys. 120, 24, 11919 (2004).

[10] C.F. Abrams, E. Vanden-Eijnden. PNAS 107, 45, 4961 (2010).

[11] P.R.L. Markwick, J.A. Mc Cammon. Phys. Chem. Chem. Phys. 13, 11, 20053 (2011).

[12] W.K. Kim, M.L. Falk. Model. Simul. Mater. Sci. Eng. 18, 34003 (2010).

[13] В.Г. Дубровский. Теория формирования эпитаксиальных наноструктур. Физматлит, М. (2009). 352 с.

[14] B. Lalmi, H. Oughaddou, H. Enriquez, A. Kara, S. Vizzini, B. Ealet, B. Aufray. Appl. Phys. Lett. 97, 223109 (2010).

[15] M.E. Davila, L. Xian, S. Cahangirov, A. Rubio, G. Le Lay. New J. Phys. 16, 95002 (2014).

[16] G.H. Vineyard. J. Phys. Chem. Solids 3, 121 (1957).

[17] P.M. Morse. Phys. Rev. 34, 57 (1929).

[18] L.A. Girifalco, V.G. Weizer. Phys. Rev. 114, 3, 687 (1959).

[19] J.F.Zigler, J.P. Biersack, U. Littmark. The Stopping and Range of Ions in Solids. Pergamon, N.Y. (1985). 321 p.

[20] H.J. Berendsen, J.P.M. Postma, W.F.V. Gunsteren, A. Di-Nola, J.R. Haak. // J. Chem. Phys. 81, 8, 3684 (1984).

[21] Л.Н. Большев, Н.В. Смирнов. Таблицы математической статистики. АН СССР, М. (1968). С. 88.

[22] Вл.В. Воеводин, С.А. Жуматий, С.И. Соболев, А.С. Антонов, П.А. Брызгалов, Д.А. Никитенко, К.С. Стефанов, Вад.В. Воеводин. Практика суперкомпьютера „Ломоносов“. Открытые системы. Издательский дом „Открытые системы“, М. 7 (2012). С. 36-39. 